Kansas State University Libraries

New Prairie Press

\title{
NONLINEAR REGRESSION PARAMETERS AS OUTCOMES: SIMPLE VS. SOPHISTICATED ANALYSES
}

Reid D. Landes

Follow this and additional works at: https://newprairiepress.org/agstatconference

Part of the Agriculture Commons, and the Applied Statistics Commons

\section{(c) (1) $\Theta($}

This work is licensed under a Creative Commons Attribution-Noncommercial-No Derivative Works 4.0 License.

\section{Recommended Citation}

Landes, Reid D. (2010). "NONLINEAR REGRESSION PARAMETERS AS OUTCOMES: SIMPLE VS.

SOPHISTICATED ANALYSES," Conference on Applied Statistics in Agriculture. https://doi.org/10.4148/

2475-7772.1068

This is brought to you for free and open access by the Conferences at New Prairie Press. It has been accepted for inclusion in Conference on Applied Statistics in Agriculture by an authorized administrator of New Prairie Press. For more information, please contact cads@k-state.edu. 


\title{
NONLINEAR REGRESSION PARAMETERS AS OUTCOMES: SIMPLE VS. SOPHISTICATED ANALYSES
}

\author{
Reid D. Landes \\ Department of Biostatistics \\ University of Arkansas for Medical Sciences \\ Little Rock, AR 72205
}

\begin{abstract}
Sometimes a nonlinear regression parameter for an individual is the outcome of interest. But due to variability among individuals, the individuals' regression parameters cannot be estimated with the same amount of precision. This problem of heterogeneous variance complicates the ultimate goal of estimating population-level regression parameters with two usual methods: (i) the simple arithmetic mean of individually estimated regression parameters and (ii) random coefficients regression (RCR). Weights are proposed for each method to account for the heterogeneity problem. The methods are illustrated with chick weights collected over time. Monte Carlo simulation allows comparison of statistical properties of the four estimators for small, moderate and large sample sizes. The arithmetic means tended to outperform the RCR estimators with respect to mean square error and bias; and their associated confidence intervals held nominal levels. Actual coverage of confidence intervals produced from RCR methods fell below nominal levels in some cases; however this discrepancy may be an algorithm error. Overall, the simpler arithmetic mean estimators tend to have either better or comparable statistical properties to those estimators from RCR methods.
\end{abstract}

\section{Introduction}

In some studies, the outcome of interest is a theoretical parameter that cannot be directly observed. Growth rates are prime examples. For instance, we can measure an individual's weight over time, but often have to assume a theoretical function which describes that individual's weight change over time. The desired growth rate is often some parameter in the function. We can estimate the growth rate for an individual given enough observations across time, but it is, alas, an estimate. Studies often focus on population parameters rather than individual ones. But a sample of individuals allows us to estimate the population. We must then sensibly combine the information from a sample of individuals to represent the population. When estimating a population parameter, we know individuals vary. If we can exactly measure an individual's value, then we only have to worry about individual-to-individual variability when estimating the population value. On the other hand, if we estimate the individuals' values, then we also need to deal with this extra source of variability. This paper proposes weights for two commonly used methods for estimating a population regression parameter

The objectives of this paper are to

(1) briefly describe commonly used statistical methods for estimating a population regression coefficient in a nonlinear model, 
(2) propose weights for individuals' data to be used in weighted versions of the methods described in (1), and

(3) evaluate the statistical properties of the usual and weighted estimators with Monte Carlo simulations.

An example motivates the objectives and provides the scenarios in the Monte Carlo simulations. A discussion of the estimator comparisons concludes the paper.

\subsection{Chick Growth}

Donnett et al. (2010) present and analyze the weights from 50 chicks that were collected from each chick every 4 weeks from Week 0 to Week 40. The same data were previously presented in Mignon-Grasteau et al. (1999) and re-analyzed in Jeffrezic et al. (2006). From an individual chicken, the weights transformed with the natural logarithm $(y)$ are assumed to follow a logarithm-transformed Gompertz growth curve,

$$
Y=m-\operatorname{dexp}\left(-x \mathrm{e}^{g}\right)+\varepsilon, \quad \text { Equation } 1
$$

with $\varepsilon$ i.i.d. $\mathrm{N}\left(0, \sigma^{2}\right)$. In this model, $m$ represents the logged mature weight, $d$ the difference between the logged birth and mature weights, $g$ the logged growth rate, and $x$ is time. Donnett et al. assume the chicks came from essentially the same population and focus on estimating the population parameters, $\mu=\mathrm{E}(m), \delta=\mathrm{E}(d)$, and $\gamma=\mathrm{E}(\mathrm{g})$. I adopt these assumptions here. Figure 1 shows the observed logged weights and fitted growth curve for a representative chick.

\section{Methods}

The following methods assume that there is a random sample of mutually independent individuals, each with a series of observations that may be fit to the same form of a regression model.

\section{$2.1 \quad$ Standard Estimators}

Simple Arithmetic Mean (SAM). The analyst fits the regression model to the data from each individual, keeping the estimated parameter of interest. (I used nonlinear least squares for estimating the regression parameters here and throughout.) The population parameter is then estimated with the simple arithmetic mean (SAM) of the individual estimates; see Chapter 3 of Diggle, Liang \& Zeger (1994). The standard error of the SAM contains both the variability in individuals' parameters and the variability in an individual's parameter estimate. A confidence interval based on a $t$-quantile enables statistical inference. Additional assumptions underlying the confidence interval are that

- the individuals each provide a complete set of data under the same schedule (i.e., same $x$ space), and

- the observations given their expected value have the same variance-covariance structure across the individuals (i.e., same $\operatorname{Var}(\boldsymbol{\varepsilon})$ ).

These two assumptions imply homogeneous variance of parameter estimates across individuals. It is worth noting that if the $x$-space is not the same across individuals (e.g., if some data are missing from some individuals), then the standard errors of regression parameters will not be the 
same even if $\operatorname{Var}(\boldsymbol{\varepsilon})$ in Equation 1 is constant across individuals. Table 1 contains the SAM estimates of $\mu, \delta$, and $\gamma$.

Figure 2 contains normal q-q plots of the individually estimated $m$ 's, $d$ 's, and $g$ 's; this figure allows visual evaluation of the normal distribution assumption. To evaluate the homogeneous variance assumption, Figure 3 has the standard errors (and their 50\% confidence intervals) of the regression parameter estimates plotted by rank order. The sample distributions of the $m$ 's and $d$ 's are likely not normal, having heavier tails than the normal. The distribution of $g$ 's comes closer to meeting the normal assumption. Since the means are of interest, samples of size 50 are likely large enough in these three cases to provide robust mean inference. From Figure 3, there also is evidence of heterogeneous variances among the estimated parameters since some pairs of standard errors were statistically different using a .05 level $F_{[9,9]}$ test for equal variances. The impact of this violation on mean inference is harder to assess, but in general, leads to intervals that are, on average, the wrong length.

Random Coefficients Regression (RCR). The observations from all of the individuals are fitted with a form of the original regression model which allows the parameters of the model to vary with the individual. Often, the RCR model assumes a normally distributed "bump”, say $g_{j}$, for individual $j$ to the population parameter, say $\gamma=\mathrm{E}(g)$. The regression models may be estimated with a variety of methods; e.g., maximum likelihood, restricted maximum likelihood, etc. I used restricted maximum likelihood implemented within the NLINMIX macro (a SAS ${ }^{\circledR}$ macro) for these examples and throughout. See Chapter 15 of Littell et al. (2006).

For the growth rate example, the RCR model is $Y=\left(\mu+m_{j}\right)-\left(\delta+d_{j}\right) \exp \left[-\chi_{j k} \exp \left(\gamma+g_{j}\right)\right]+\varepsilon_{j k} . Y_{j k}$ is the logged weight for chick $j$ at week $x_{j k}$. The random vector $(m, d, g)_{j}$ is assumed normal with a zero mean and general covariance and to be independent of $\varepsilon_{j k}$ which is also assumed normal with a zero mean. See Table 1 for point and interval estimates from the RCR model.

Table 1. Point and 95\% confidence intervals for population-level nonlinear regression parameters from four methods: SAM - simple arithmetic mean; WAM - weighted arithmetic mean; RCR - random coefficients regression; WRCR - weighted RCR.

\begin{tabular}{l|c|c|c|c|c|c|} 
& \multicolumn{2}{|c|}{$\boldsymbol{\mu}$} & \multicolumn{2}{|c}{$\boldsymbol{\delta}$} & \multicolumn{2}{c}{$\boldsymbol{\delta}$} \\
Method & Estimate & CI & Estimate & CI & Estimate & CI \\
\hline SAM & 7.772 & $(7.699,7.844)$ & 4.164 & $(4.101,4.227)$ & -1.849 & $(-1.908,-1.791)$ \\
WAM & 7.770 & $(7.698,7.843)$ & 4.162 & $(4.099,4.225)$ & -1.848 & $(-1.906,-1.789)$ \\
RCR & 7.756 & $(7.686,7.826)$ & 4.139 & $(4.077,4.200)$ & -1.855 & $(-1.913,-1.798)$ \\
WRCR & 7.755 & $(7.685,7.825)$ & 4.147 & $(4.085,4.208)$ & -1.852 & $(-1.910,-1.795)$
\end{tabular}

\subsection{Weighted Estimators}

When analyzing the data for each individual chick, the standard errors for the parameter estimates ranged from .0138 to .0607 among the $m$ 's, .0317 to .1108 among the $d$ 's, and .0168 to .0564 among the $g$ 's. At best, the variances have an 11-fold difference and at worst a 20 -fold 
difference. Weighting the data used in drawing inference on population regression parameters is an effective way to account for the heterogeneous variances in the individuals' regression parameter estimates.

Weighted Arithmetic Mean (WAM). For arithmetic mean estimators, I constructed a "heterogeneity variance" weight as described in Sidik and Jonkman (2005) for the estimates of a particular regression parameter. The weight for, say $g_{j}$, is closely akin to $\left(s^{2}{ }_{g}+\text { s.e. }\left(g_{j}\right)^{2}\right)^{-1}$, where $s^{2}$ is the sample variance of the estimated $g$ 's. These weights, along with their associated estimates, were then used in computing a weighted arithmetic mean (WAM) and confidence interval for population regression parameter of interest. Table 1 has the WAM point and interval estimates.

Weighted Random Coefficients Regression (WRCR). For the RCR estimators, the weight used for all the observations from a single individual was the trace of the precision matrix of the regression parameter estimators. This weight was then included in the RCR to produce weighted point and interval estimates of the population regression parameters. See Table 1 for WRCR estimates of $\mu, \delta$, and $\gamma$.

\subsection{Monte Carlo Experiment}

I conducted a Monte Carlo experiment mimicking the data and their assumed model from the chick growth example. To investigate the effect of sample size on the estimators' performance, I generated samples of $N=15,30$, and 60 individuals. The Monte Carlo experiment was conducted as follows.

(1) Generated an individual's dataset.

a. Generated $m \sim \mathrm{N}\left(7.77,0.22^{2}\right), d \sim \mathrm{N}\left(4.16,0.25^{2}\right)$, and $g \sim \mathrm{N}\left(-1.85,0.21^{2}\right)$ where parameters of $\mathrm{N}(*, *)$ were estimated from the example data.

b. Randomly drew $\sigma_{\mathrm{y}}{ }^{2}$ from the 50 observed regression means square errors after fitting Equation 1 to the weights from the 50 chicks. These $\sigma_{\mathrm{y}}{ }^{2}$ ranged from $9.72 \times 10^{-4}$ to $1.16 \times 10^{-2}$.

c. Generated $\mathrm{Y} \sim \mathrm{N}\left(\mathrm{f}(x ; m, d, g), \sigma_{\mathrm{y}}{ }^{2}\right)$, with $x$ the same as in the example, and $\mathrm{f}(*)$ the mean portion of Equation 1.

(2) Repeated step 1 for $N$ individuals.

(3) Estimated $\mu, \delta$, and $\gamma$ with the proposed methods.

(4) Repeated previous steps 1000 times.

The statistical properties of interest were bias, mean square error (MSE), and coverage probability of nominal 95\% CIs. Within each sample size $(N)$, the bias for an estimator was computed as the mean difference between the estimate and the true value, averaged over the 1000 generated datasets. MSE was the mean squared difference between the estimate and true value, averaged over the 1000 generated datasets. Coverage probabilities were the fraction of 1000 interval estimates (having 95\% confidence) containing the true value. 


\section{$3 \quad$ Results}

Mean square errors (MSEs) for the estimators of the three population parameters, $\mu, \delta$, and $\gamma$, are respectively found in Figures $4-6$, and are paneled by sample size $(N)$. For two of the parameters, $\mu$ and $\delta$, the simple methods have notably lower MSEs than the more sophisticated methods. These differences between the simple and sophisticated methods appear to increase with sample size (see Figures 4 and 5). But for the growth rate parameter, $\gamma$, the sophisticated methods slightly edge the simpler methods in MSE performance (see Figure 6), with no clear pattern in these differences as the sample size changes. Comparing between the two simple methods and between the two sophisticated methods, weighted versions tend to beat the unweighted versions; however, the improvements are practically negligible for each of the parameters.

Figures $7-9$ respectively contain bias, expressed as percent relative to the true value, paneled by $N$ for the estimators of the three population parameters, $\mu, \delta$, and $\gamma$. As with MSE, the arithmetic mean estimators of $\mu$ and $\delta$ had substantially less bias than the RCR estimators (see Figures 7 and 8). For both parameters, the bias in the simple estimators was practically negligible $(\leq$ $0.05 \%$ ) regardless of sample size. For the sophisticated estimators, bias was less than $0.66 \%$ for the smallest sample size examined, and improved as $N$ increased. When estimating the growth rate, $\gamma$, there was little practical difference in bias among the four estimators (see Figure 9). Though bias for each of the $\gamma$ estimators was greatest for the $N=15$ case, it did not exceed $0.20 \%$ of the true $\gamma$ value. For all estimators, estimates of $\gamma$ moved closer to the truth on average as the sample sizes increased. Finally, for all the estimators studied here, bias was essentially negligible with no estimated bias exceeding $\pm 0.7 \%$ of the true value.

Coverage probabilities of interval estimators (having nominal confidence of 95\%) are paneled by $N$ in Figures $10-12$ for $\mu, \delta$, and $\gamma$, respectively. Again, for the parameters $\mu$ and $\delta$, the interval estimators from the RCR methods did not perform as well as those based on arithmetic means. The simple estimators tended to hold the nominal level across the sample sizes investigated. Interval estimates coming from RCR analyses were closest to the nominal level for both $\mu$ and $\delta$ when $N=15$ (between .935 and .938), but deteriorated as $N$ increased (see Figures 10 and 11). For the growth rate, $\gamma$, coverage probabilities tended to be at or close to $95 \%$ regardless of estimation method or sample size (see Figure 12).

\section{Discussion}

Both arithmetic means and random coefficients regressions have their appeal to the practioner. Arithmetic means are simple to understand, and can also be readily computed by nonstatisticians. Random coefficients regressions (RCRs) allow the statistically savvy to fit theoretical models to the data. But both methods also have their drawbacks. RCRs can be difficult to fit, have more assumptions that are more difficult to evaluate than those for simpler methods, and are not well-understood by those with little statistics background. Arithmetic 
means can fail to address certain aspects of the data (like the related nature of the parameter estimates coming from the same individual) and are often not as efficient as more sophisticated methods. In this paper, I compared the statistical properties of the estimators from simple and sophisticated methods to better understand which type of these two methods may be more appropriate in nonlinear models. Since both methods often assume homogeneous variances, I also included weighted versions of each to account for violations of this assumption. Further, since estimation of population parameters was the central goal, I chose to consider increases in the number of individuals rather than increasing the number of observations from an individual. The relevant variability to population parameter estimation is the variability among individuals. Increasing the number of observations taken within an individual increases the precision with which we know an individual's parameters, and would asymptotically drive the weights for each person to the same value.

The comparisons among the methods, via Monte Carlo simulation, were done for each of the parameters in the example nonlinear model. The patterns in the results among the three parameters were not consistent. In particular, for $\mu$ and $\delta$, the arithmetic mean estimators outperformed RCR estimators. For $\gamma$, however, RCR estimators were just as good as arithmetic mean estimators. Since the RCR estimators did not conclusively beat the arithmetic estimators on any of the parameters, this simulation study points to arithmetic means as being the preferred method.

When generating data in the Monte Carlo experiment, Step 1b induced heterogeneous variances among the individuals. The proposed weights did tend improve estimation properties within each type of estimator, arithmetic mean and RCR; however, these improvements were negligible. I suspect the advantage of the weighted version of the estimators over their unweighted analogues increases as heterogeneity in the variances increases.

One result from the Monte Carlo experiment that goes against intuition is the decrease in actual coverage probabilities of RCR interval estimators as the sample size increased. This is especially concerning since the bias of the associated RCR point estimators decreased with sample size increases. At this point in time, it is unknown whether the standard errors become increasingly optimistic as the sample size increases or the error degrees of freedom associated with the confidence intervals are too large or some other reason. In order to determine under what conditions this statistically anomaly in coverage probabilities appears, I conducted a series of simulation studies. I briefly report on them here.

For each case in Table 2, I generated data for individual $j=1,2, \ldots, N$ according to the indicated scenario with $x=0,4,8, \ldots, 40$. Sample sizes $(N)$ investigated were $15,30,60$, and 250 . At least 500 data sets per case $\times N$ combination were generated and analyzed with the RCR and WRCR methods implemented in the NLINMIX macro. Cases 1 and 2 are essentially random coefficients linear regressions. No problems were noted. The problem of decreasing coverage probabilities with increasing sample size was found in Case 3, a random coefficients nonlinear regression with homogeneous variance. Case 3 is a "tamer" version of the main Monte Carlo experiment which allowed the variance of $\varepsilon$ to be heterogeneous among the individuals. 
Table 2: A series of simulation studies under 3 scenarios, with each series examining at least 500 Monte Carlo simulated data sets for $N=15$, 30, 60, and 250.

\begin{tabular}{ccl} 
Case & \multicolumn{1}{c}{ Scenario } & \multicolumn{1}{c}{ Main Result } \\
\hline 1 & $Y=7.77-d_{j} \exp \left(-\chi \mathrm{e}^{(-1.85)}\right)+\varepsilon$ & As $N$ increased, coverage probabilities held nominal \\
& $\varepsilon \sim \mathrm{N}(0, .00377)$ & levels for interval estimators of $\delta$. \\
& $d_{j} \sim \mathrm{N}\left(4.16, .25^{2}\right)$ & \\
\hline 2 & $Y=m_{j}-d_{j} \exp \left(-\chi \mathrm{e}^{(-1.85)}\right)+\varepsilon$ & As $N$ increased, coverage probabilities held nominal \\
& $\varepsilon$ and $d_{j}$ as in Case 1 & levels for interval estimators of $\mu$ and $\delta$. \\
& $m_{j} \sim \mathrm{N}\left(7.77, .22^{2}\right)$ & \\
\hline 3 & $Y=m_{j}-d_{j} \exp \left(-\chi \exp \left(g_{j}\right)\right)+\varepsilon$ & As $N$ increased, coverage probabilities dropped well \\
& $\varepsilon, d_{j}$ and $m_{j}$ as in Case 2 & below nominal levels for interval estimators of $\mu$ and \\
& $g_{j} \sim \mathrm{N}\left(-1.85, .21^{2}\right)$ & $\delta$, but held nominal levels for interval estimators of $\gamma$.
\end{tabular}

The NLINMIX macro was written to handle models such as Case 3, so I was surprised to see this coverage probability problem appear in this "simple" random coefficients nonlinear regression. With $N=250$, the coverage probabilities of $95 \%$ CIs for $\mu$ and $\delta$ were 0.777 and 0.715 , respectively. Using the same generated data for Case 3 with $N=250$, I fitted the (data-generating) RCR model with the nlme() function in R version 2.11.1 as was fitted in the NLINMIX macro. The R-produced $95 \%$ CIs for $\mu$ and $\delta$ covered the true values $95.7 \%$ and $96.2 \%$ of the time. The results from $\mathrm{R}$ suggest that there is likely an algorithm error in the NLINMIX macro.

With the R results of Case 3 with $\mathrm{N}=250$ in hand, I also compared the MSE of RCR estimators with that from the SAM and WAM estimators. The RCR estimators (from R) tended to have lower MSE than those from SAM or WAM, but not substantially so. Across the three parameters ( $\mu, \delta$, and $\gamma$ ), the MSE from the RCR estimator was, at best, $0.5 \%$ smaller than the MSE from either a SAM or WAM estimator.

This study has uncovered an apparent bug in the NLINMIX macro that needs to be addressed. Unfortunately, the bug renders the comparisons between the simple and sophisticated methods inconclusive. From this point, all of the random coefficients regressions should be re-fitted with either the nlme() function in R, a different procedure or macro in SAS, or some other suitable software. Once the results from these analyses have been obtained, the comparisons between the simple and sophisticated methods can be made. Any reader wanting the SAS and R code used perform this study may contact me at rdlandes@gmail.com. 


\section{References}

Diggle, P.J., Liang, K.-Y., and Zeger, S.L. (1994). Analysis of longitudinal data. Oxford University Press, USA. 272 pages.

Donnet, S., Foulley, J.-L., and Samson, A. (2010). Bayesian analysis of growth curves using mixed models defined by stochastic differential equations. Biometrics 66, 733-741.

Jaffrezic, F., Meza, C., Lavielle, M., and Foulley, J. (2006). Genetic analysis of growth curves using the SAEM algorithm. Genetics Selection Evolution 38, 583-600.

Littell, R.C., Milliken, G.A., Stroup, W.W., Wolfinger, R.D., and Schabenberger, O. (2006). SAS ${ }^{\circledR}$ for Mixed Models, $2^{\text {nd }}$ Edition. SAS Institute, Inc., Cary, NC. 813 pages.

Mignon-Grasteau, S., Beaumont, C., LeBihan, E., Poivey, J., de Rochambeau, H., and Ricard, F. (1999). Genetic parameters of growth curve parameters in male and female chickens. British Poultry Science 40, 44-51.

Sidik, K. and Jonkman, J.N. (2005). Simple heterogeneity variance estimation for meta-analysis. Applied Statistics 54, 367-384. 


\section{Chick Growth}

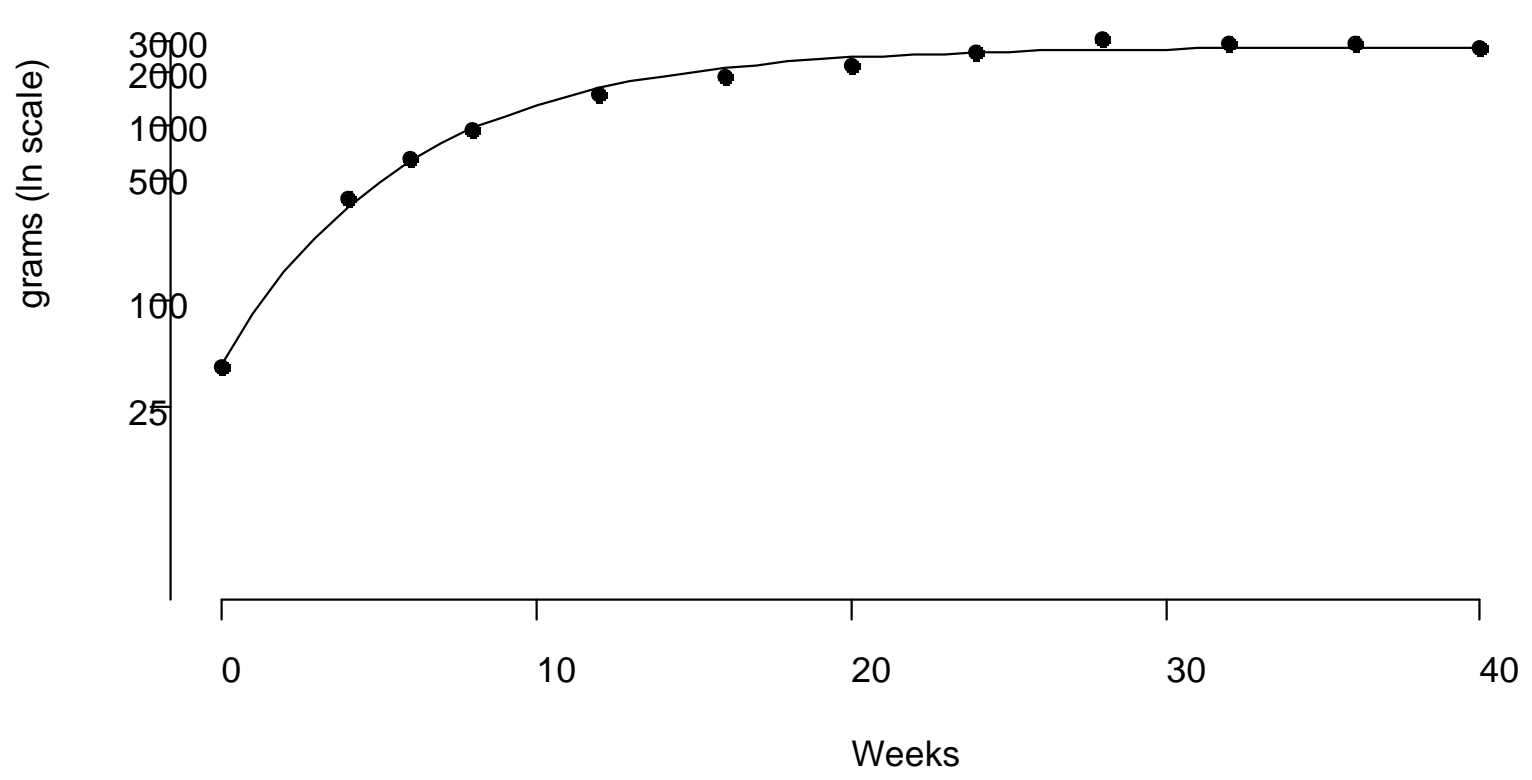

Figure 1. Representative chick weights, transformed with the natural logarithm, plotted over time and fitted with a logarithm-transformed version of the Gompertz model (Equation 1). 

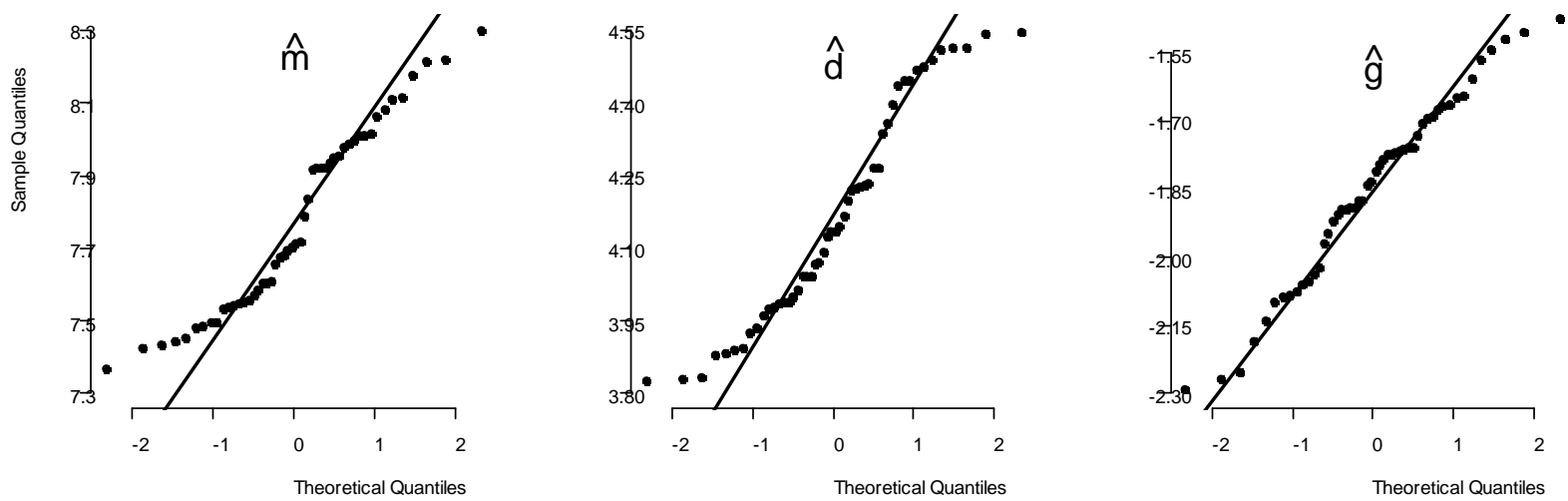

Figure 2. Normal q-q plots of nonlinear regression parameter estimates obtained from fitting log-transformed weights with Equation 1 to each of 50 chicks.
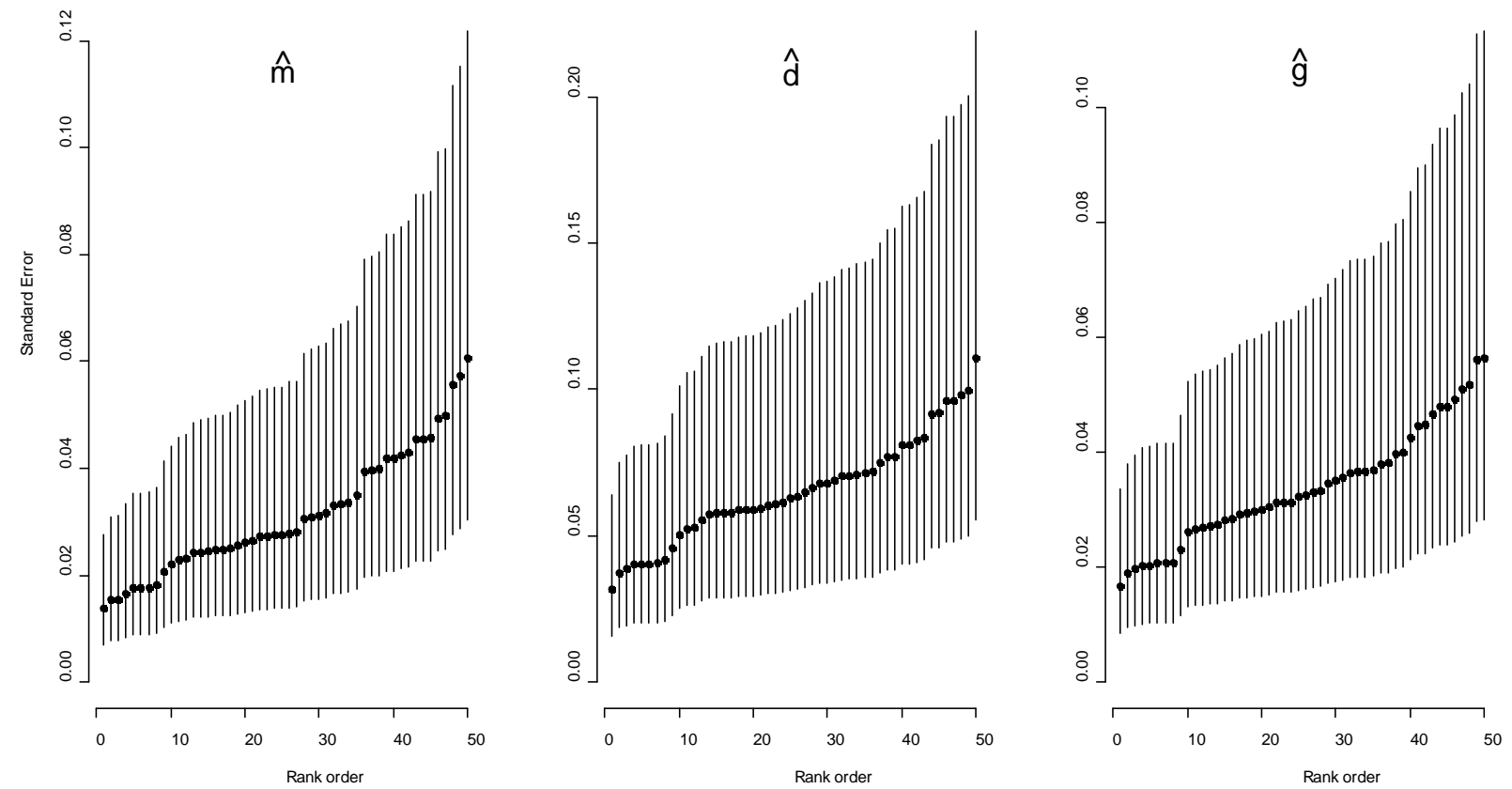

Figure 3. Plotted by rank order, standard errors of nonlinear regression parameter estimates obtained from fitting log-transformed weights with Equation 1 to each of 50 chicks. Error bars depict the range of standard errors that are not statistically different at the .05 level of a $F_{[9,9]}$ test for equal variances. 

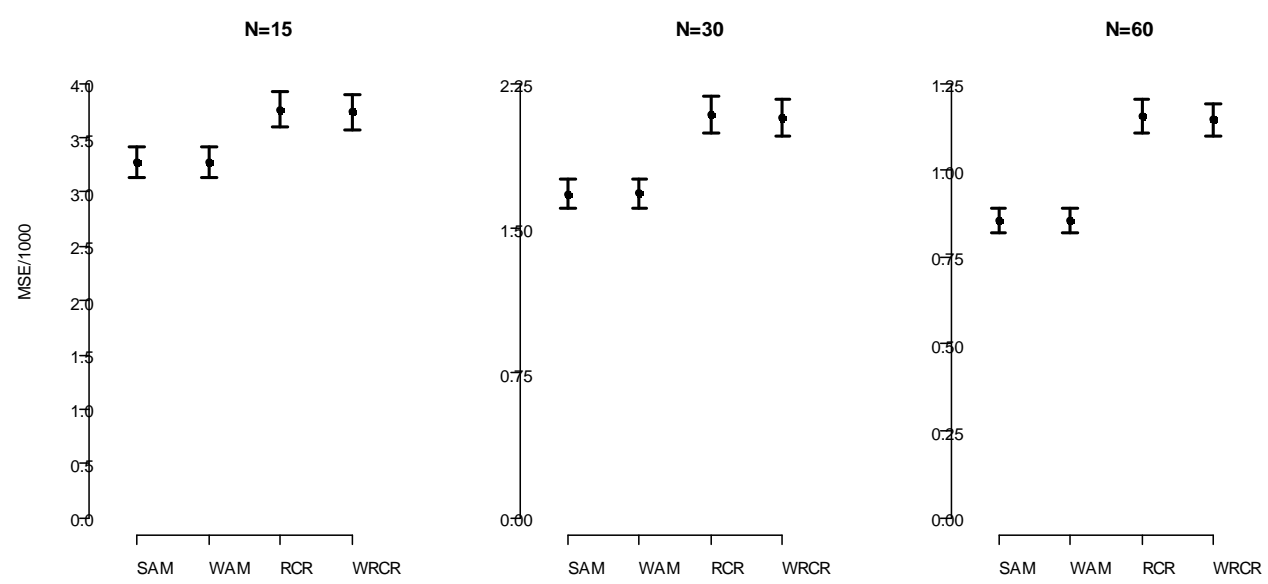

Figure 4: Mean square error (MSE) for four estimators of $\mu$. Bars are Monte Carlo error.
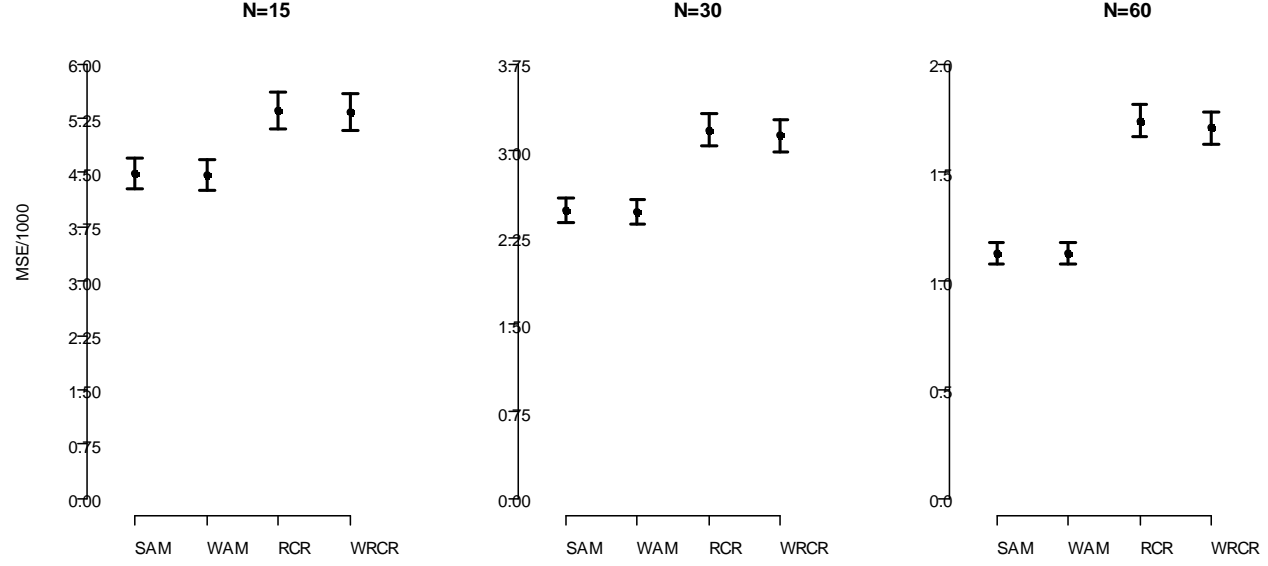

Figure 5: Mean square error (MSE) for four estimators of $\delta$. Bars are Monte Carlo error.
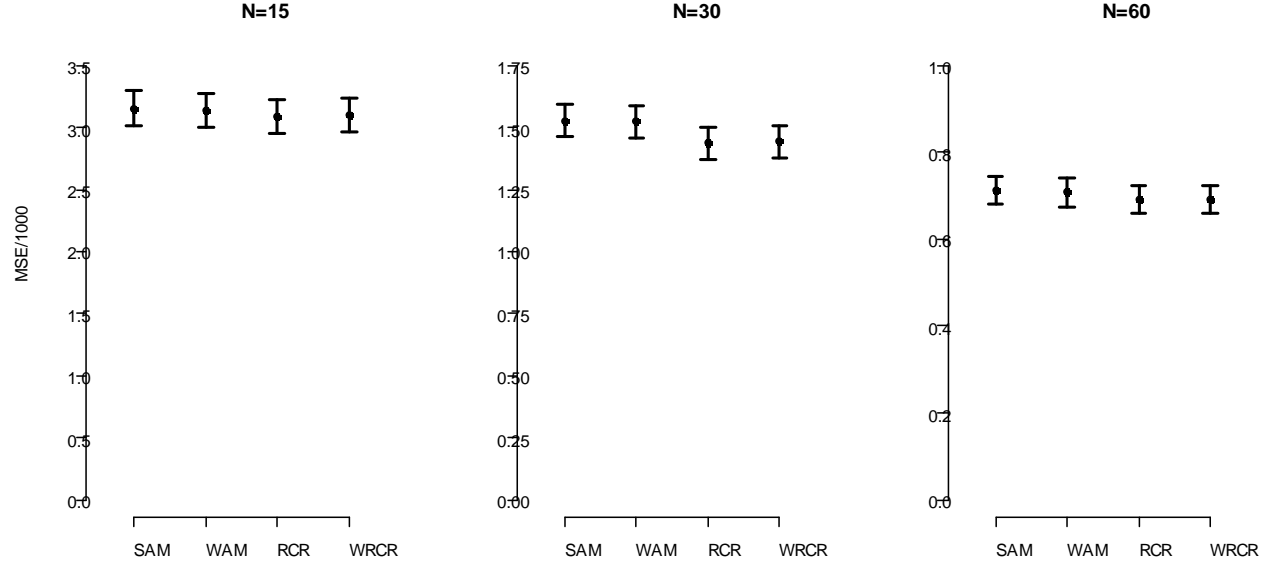

Figure 6: Mean square error (MSE) for four estimators of $\gamma$. Bars are Monte Carlo error. 


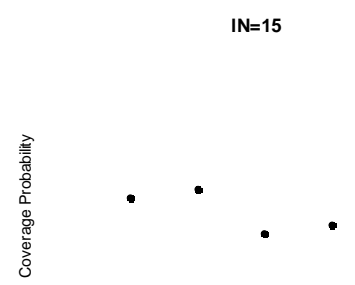

